\title{
ANÁLISIS DE LA
}
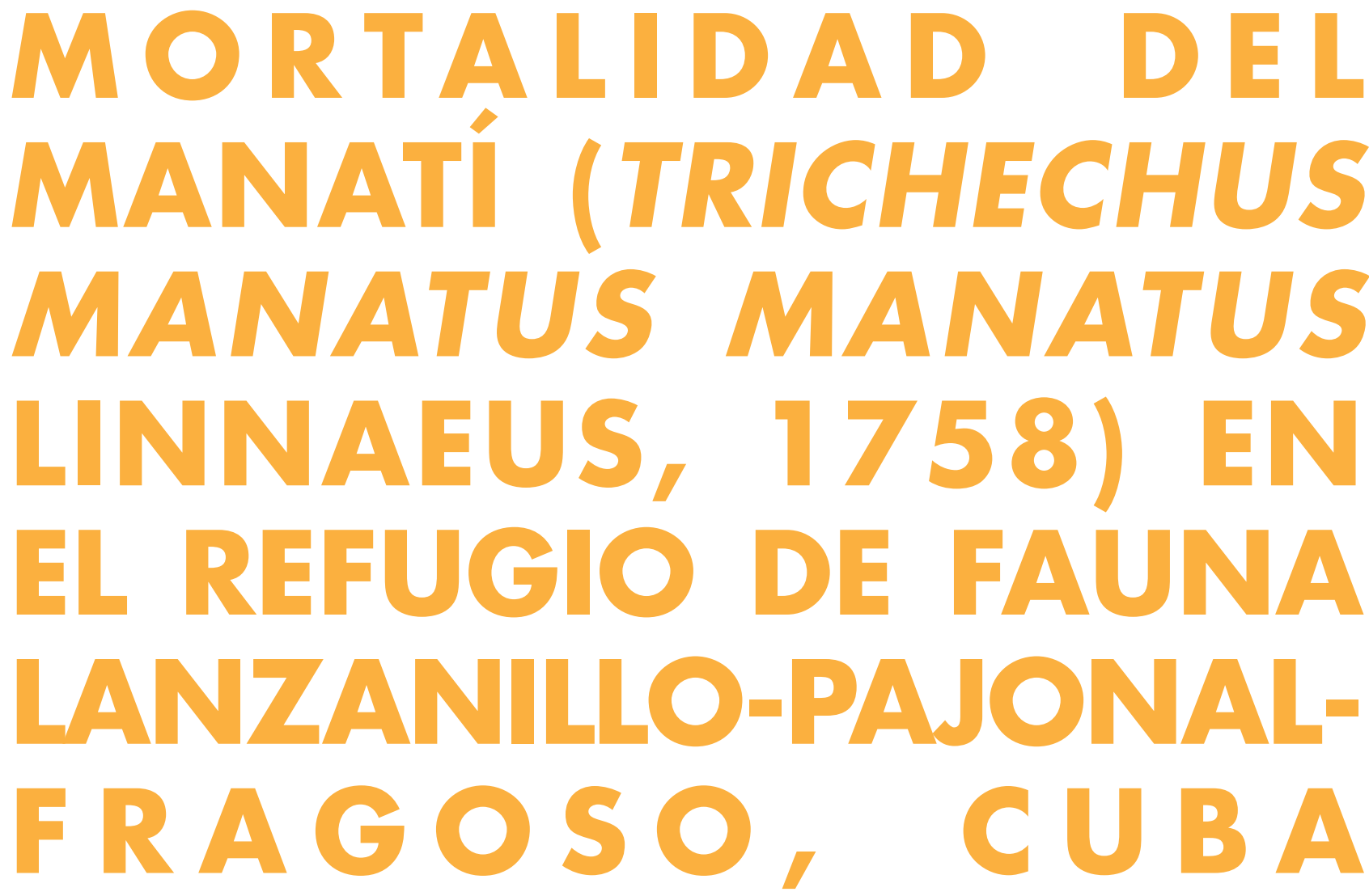

\section{E. García Alfonso ${ }^{1}$ y R. Rodríguez González $z^{2}$}

i Refugio de Fauna Lanzanillo-Pajonal-Fragoso. Empresa Nacional para la Protección de la Flora y la Fauna, Villa Clara. (ENPFF)

2 Departamento de Biología, Universidad Central Marta Abreu de las Villas (UClV) RENERG@UCLV.EDU.CU

\section{RESUMEN}

El refugio de Fauna Lanzanillo-Pajonal-Fragoso, es un Área Protegida con 76.490 Ha marinas y su territorio terrestre encierra la Bahía de Nazabal, con condiciones naturales para mantener poblaciones de manatíes ya que presenta fondos con vegetación que constituyen su dieta. Además a la Bahía desembocan ríos que sirven de abrevaderos de agua dulce. Se tomaron datos de 12 adultos y una cría muertos entre los años 1987-2008, realizando recorridos por toda la costa. Se confeccionó una base de datos de cada ejemplar y se determinó que el chinchorreo como arte de pesca estaba afectando la especie. Se declaró la Bahía de Nazabal como Zona Protegida o bajo Régimen Especial de Uso y Protección, decretado por la Secretaria de la Comisión Consultiva de Pesca. El chinchorreo en la bahía permanece todavía en un sector donde hasta la fecha no se han registrado más muertes. 
Palabras clave: Manatí, mortalidad, Trichechus manatus manatus, área protegida.

\section{ABSTRACT}

Lanzanillo Pajonal-Fragoso Animals Shelter is a protected marine area with $76,490 \mathrm{Ha}$ and in its land territory is located Nazabal Bay, with natural conditions to maintain manatees due to it has see beds vegetation that provide the diet as well as rivers that flow to the Bay and serve as drinking fresh water. There were collected data of dead individuals including 12 adults and a baby from 1987-2008, doing patrols along the coast. To determine possible causes of death it was taken into account that the fishing gear coincided with the stranding. It was drawn up a database of each analyzed specimen and it was determined that the chinchorreo gear was affecting the species. Nazabal Bay was declared a protected or under special use and protection area by the Secretary of the Advisory Committee on Fisheries. The chinchorreo gear in the bay was not completely removed, remaining a sector where that art of fishing is still practiced up to the present there have not caused more deaths.

Key words: Manatees, mortality, Trichechus manatus manatus, protected area.

\section{INTRODUCCIÓN}

El Área Protegida Lanzanillo-Pajonal-Fragoso, con categoría de Manejo Refugio de Fauna, consiste en un territorio marino costero de forma alargada, estrecha y orientada de este a oeste y abarca una superficie de $87.070 \mathrm{Ha}(76.490 \mathrm{Ha}$ marinas y $10.580 \mathrm{Ha}$ terrestres), que incluye acuatorios sobre la plataforma insular, numerosos cayos y cayuelos, donde las tierras emergidas son evidentemente minoritarias. Posee una alta diversidad de hábitat, los más significativos son los manglares de desembocadura (en ríos, arroyos y canales) costeros, de canales entre cayos y de cayos, tales combinaciones ambientales favorecen una notable gama de hábitat que se refleja en la diversidad de la flora y la fauna.

El manatí de las Antillas (Trichechus manatus manatus) se distribuye desde el Estado de Tabasco en México hasta Recife en Brasil (LEFEBRE et al,
1989), se encuentra además en Las Antillas Mayores incluyendo Cuba, Jamaica, Puerto Rico, La Espańola y pequeños reductos en las Antillas Menores (DOMNING \& HAYEK, 1986).

Debido a las diversas amenazas a las que se enfrenta la especie se encuentra catalogada en el Libro Rojo de la UICN, bajo la categoría de especie vulnerable y también se encuentra en el apéndice I de CITES, inciso b (RATHBUN et al, 1985, LEFEBVRE et al. 1989).

Los varamientos se clasifican en dos tipos: los varamientos individuales que involucran a un sólo animal o a una madre con su cría, y los varamientos masivos, que involucran dos o más animales de la misma especie, exceptuando el caso de una madre con su cría. (GERACI \& LOUNSBURY, 1993).

Es necesario tener presente que los varamientos individuales o masivos son eventos inesperados que consecuentemente puede conducir a la mortandaz de cualquier especie marina y que requiere de una respuesta inmediata para reducir su probabilidad de muerte (WIIKINSON, 1996).

El objetivo de este trabajo es determinar las posibles causas que están provocando la muerte de esta especie en la Bahía de. Para ello se registró y estudió del varamiento de estos mamíferos marinos con el fin de proporcionar información importante para el manejo de los ecosistemas costeros.

\section{DESCRIPCIÓN DEL ÁREA DE TRABAJO}

El área de trabajo es un sistema manglar-seibadalarrecife que por su productividad y posibilidades de refugio ofrece una garantía de soporte de la vida. Estos hábitat constituyen sitios típicos de reproducción, reclutamiento o cría de numerosas especies debido a su alta productividad.

El Refugio de Fauna exhibe una alta diversidad de hábitat (ALCOLADO, 2007), compuesta por manglares de desembocadura (en ríos, arroyos y canales), costeros, de canales entre cayos. Los seibadales se asientan sobre cieno, grava, arena y cieno-arena, los fondos duros aparecen mayoritariamente en la zona norte exterior caracterizada por especies vegetales como Halodule wrighti, Syringodium filiforme, Thalassia testudinum, Ruppia maritima. Esta elevada diversidad de hábitat se refleja en la diversidad de 
la flora y la fauna, donde especies de requerimientos muy específicos para la reproducción, como el Manatí (Trichechus manatus) tienen lugar para reproducirse.

Se realizaron recorridos de observación en busca de animales varados entre los años 1987-2008, por la costa que bordea la Bahía de Nazabal, estableciendo un sistema de información a través del personal que transita por el área conformado por los trabajadores de la Estación Biológica Manatí, pescadores deportivos, tropas guardafronteras y cuerpo de guardabosque. Se realizaron tomas de muestras y necropsias para determinar la posible causa de muerte a los animales que su estado lo permitió.

\section{RESULTADOS Y DISCUSIÓN}

Se realizó el análisis de 13 cadáveres de manatíes (12 adultos y una cría), encontrados en el Refugio de Fauna Lanzanillo-Pajonal-Fragoso, entre los ańos 1987 y 2008 (Figura 1). La mayoría de los varamientos ocurrieron en la bahía de Nazabal o en zonas cercanas a esta.

De los 13 animales varados 12 fueron encontrados entre los años 2001 y 2008 (Figura 1), lo que pudiera deberse a una mayor participación de los pobladores en la detección y comunicación de eventos de esta índole. Además en estos años se incrementó en el área de estudio el arte de pesca por chinchorreo que consiste en arrastrar mayas de pesca de menor diámetro de abertura de la misma, por el fondo de la bahía, colectando las presas en un embudo final o matadero. Esto pudo causar la alta mortalidad reportada entre los años 2006 y 2008, donde los manatíes eran víctimas de estos artes de pesca.

Los manatíes son animales que nadan lentamente (5-7 $\mathrm{km}$ por hora) a su vez, su cuerpo cilíndrico y de gran diámetro junto a su poca destreza hace que caigan fácilmente en las redes (MIGNUCIIGIANONI, 1990, MIGNUCII-GIANONI et al, 1991).

Los manatíes son mamíferos de comportamiento tímido, cuya estrategia para defenderse es huir y esconderse (FREEMAN \& QUINTERO, 1990). Es de esperar que en áreas con excesivo movimiento antrópico, bien por tránsito de botes y por actividad de pesca, los manatíes tiendan a huir o por lo menos a disminuir su actividad (YOUNG et al, 1993). Al igual que todos los mamíferos, el manatí

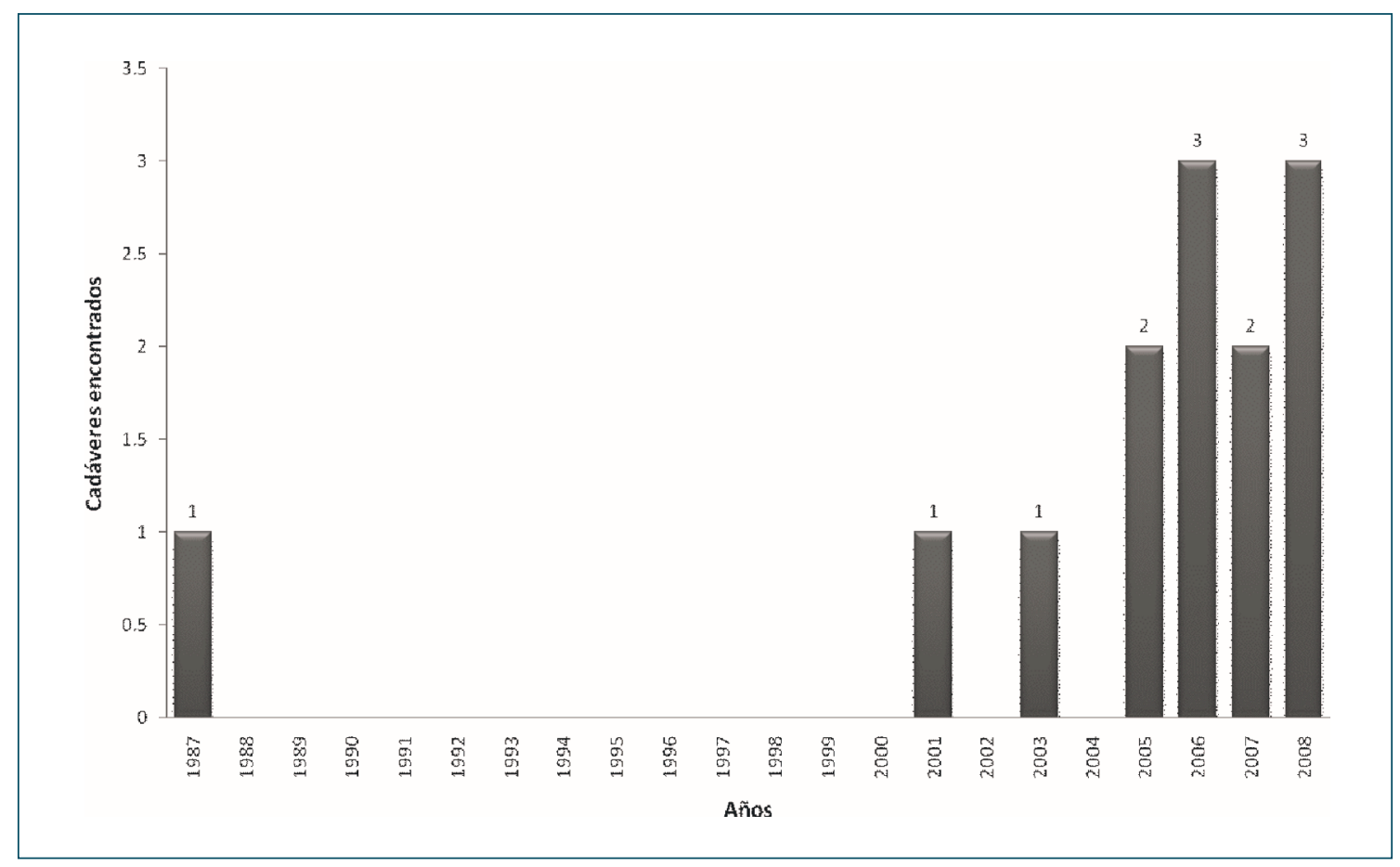

Figura 1. Cadáveres de manatí (Trichechus manatus manatus) encontrados en el área de estudio entre los años 1987 y 2008 
posee respiración pulmonar, por lo que cada dos o tres minutos emerge a la superficie para respirar, pudiendo realizar apneas de hasta 20 minutos, esto los hace vulnerables a las embarcaciones pesqueras que pueden ocasionarles la muerte al impactar contra su cuerpo (NACIONAL MARINE FISHERIES SERVIE, 1992).

Los resultados muestran que la actividad pesquera podría tener gran impacto en las poblaciones de manaties, siendo importante controlar un posible aumento en la actividad antrópica local, que conllevaría al desplazamiento de los manatíes del interior de la Bahía de Nazabal.

Se les observa fácilmente en grupos de dos a cuatro animales durante la actividad de apareamiento en los sitios donde se concentra el alimento, y en el invierno en las aguas cálidas cercanas a las desembocaduras de ríos, siendo las muertes más frecuentes en los primeros meses de cada año de estudio en estas zonas (Figura 2).
Los cadáveres de manatíes se encontraron fundamentalmente en cuatro estados de descomposición, los cuales fueron, huesos secos, animales en avanzado estado de descomposición, animales moderadamente descompuestos, y cadáveres frescos. Algunos de los manatíes se encontraron desmembrados e incluso con heridas punzantes lo que indica que la carne fue usada para el consumo humano, como muestra la Figura 3. Se registran muertes por ahogamiento, a pesar de que en algunos animales, por su estado, no fue posible determinar la causa exacta. Se tomaron muestras de material óseo, músculo etc, para futuros estudios genéticos y ecotoxicológicos así como para la determinación de la edad de los animales.

Las causas que pudieran estar causando directamente la muerte de los individuos de la población que vive el la Bahía Nazabal, son la pesca con chinchorreo y el excesivo trasporte de embarcaciones pesqueras en el área.

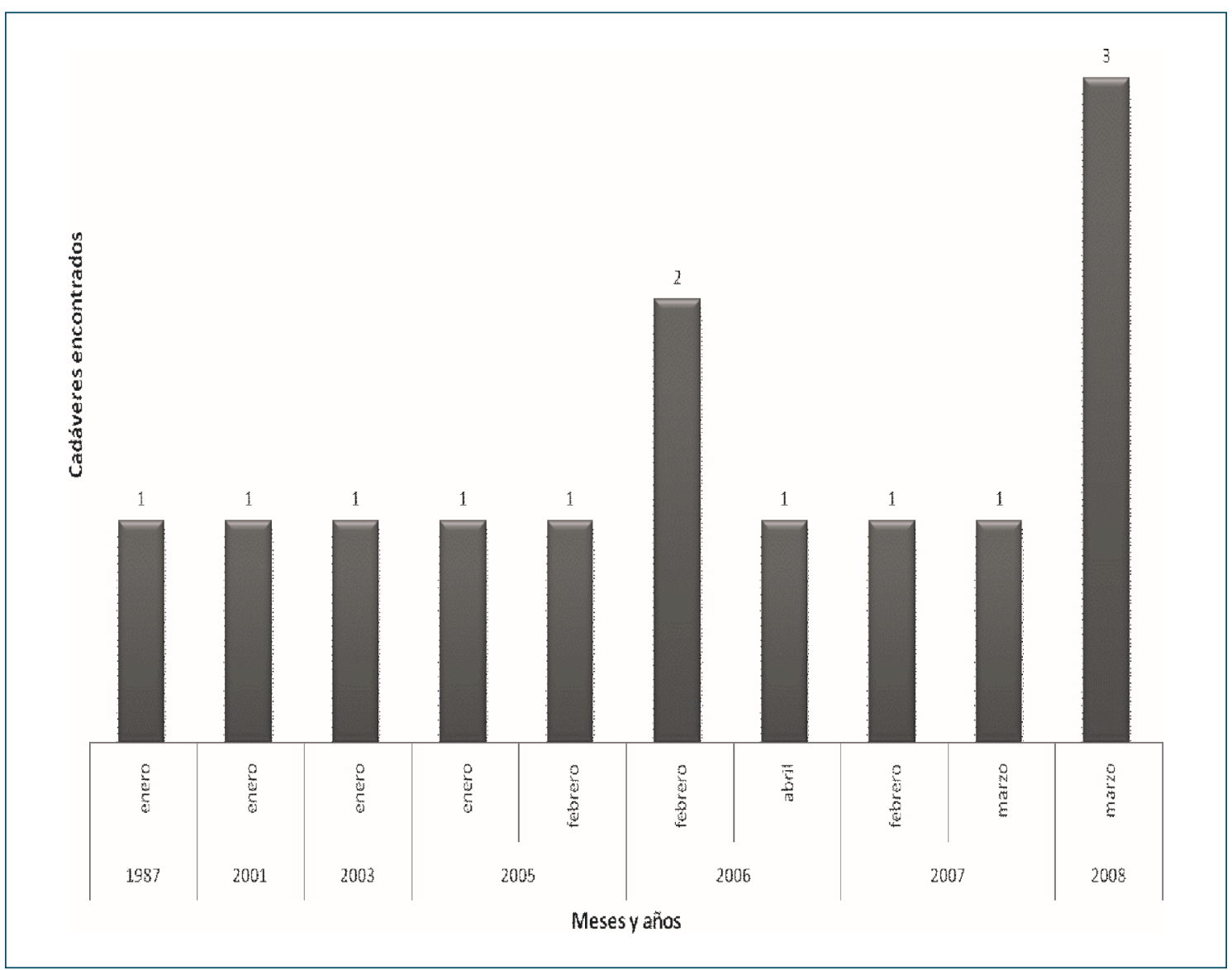

Figura 2. Cadáveres por meses en los años estudiados. 
Debido a las abundantes muertes de esta especie de importancia biológica y ecológica que tiene esta especie para la bahía y a raíz del presente estudio, se creó en la Bahía de Nazabal una Micro-estación Biológica, dentro de la Estación Biológica Manatí, perteneciente a la Empresa Nacional para Protección de la Flora y la Fauna, con el objetivo de fortalecer las acciones de conservación y protección del manatí, así como promover la educación ambiental y realizar estudios de abundancia, distribución y análisis de la mortalidad en dicha bahía. Se declaró la Bahía de Nazabal como Zona Protegida o bajo Régimen Especial de Uso y Protección, decretado por la Secretaria de la Comisión Consultiva de Pesca. El chinchorreo en la bahía se eliminó casi completamente, quedando un sector donde aun se realiza este arte de pesca, si bien, hasta la fecha no han aparecido más muertes.

Estos estudios de mortalidad de manatíes han servido como puente para un entendimiento entre diferentes usuarios de estas áreas, lo que permitirá un mejor manejo de la misma con la finalidad de garantizar la supervivencia del manatí.

La conservación del Trichechus manatus manatus requiere una preservación del ambiente como un todo (MIGNUCCI-GIANNONI et al 1993), incluyendo otras especies que comparten el hábitat con el manatí. Debe continuar el apoyo a la investigación científica de ésta y otras especies en el Área Protegida Lanzanillo-Pajonal-Fragoso, con miras a la valoración cultural y conservación de nuestros recursos.

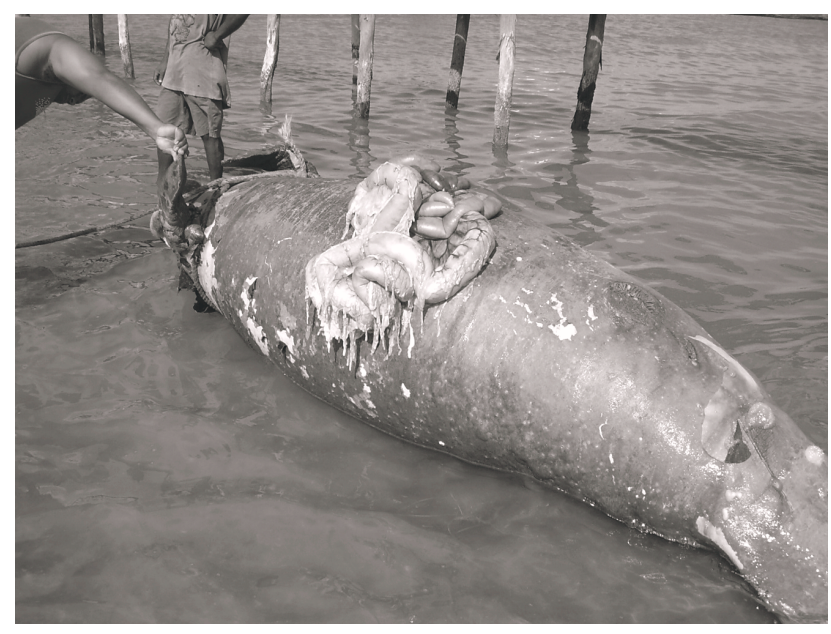

\section{REFERENCIAS}

ALCOLADO, M. P. 2007. Ecosistema SabanaCamaguey. Estado actual, avances y desafio en la protección y uso sostenible de la biodiversidad. Proyecto PNUD. Editoial Academia 182 pp.

DOMNING, D.P. \& L.C. HAYEK. 1986. Interspecific and intraspecific morphological variation in manatees (Sirenia: Trichechus). Mar. Mamm. Sci. 2:87-144.

FREEMAN, J. \& H. QUINTERO. 1990. The distribution of West Indian manatees (Trichechus manatus) in Puerto Rico: 1988-1989. NTIS PB91-137240. Springfield, VA. 43 pp.

LEFEBVRE, L.W., T.J. O'SHEA, G.B. RATHBUN \& R.C. BEST. 1989. Distribution, status, and biogeography of the West Indian manatee. Biogeography of the West Indies, 567-610.

NATIONAL MARINE FISHERIES SERVICE. 1992. Proposed Regime to Govern Interactions Between Marine Mammals and Commercial Fishing Operations. Silver Spring, MD. 96 pp.

RATHBUN, G.B., CARR, N., CARR, T., \& C.A. WOODS. 1985. The distribution of manatees and sea turtles in Puerto Rico, with emphasis on Roosevelt Roads Naval Station. NTIS PB 85-151847 AS. Springfield, VA. 83 pp.

YOUNG, N.M., S. IUDICELLO, K. EVANS, \& D. BAUR. 1993. The incidental capture of marine mammals in U.S. fisheries: problems and solutions. Center for Marine Conservation, Washington, D.C. 415 pp.

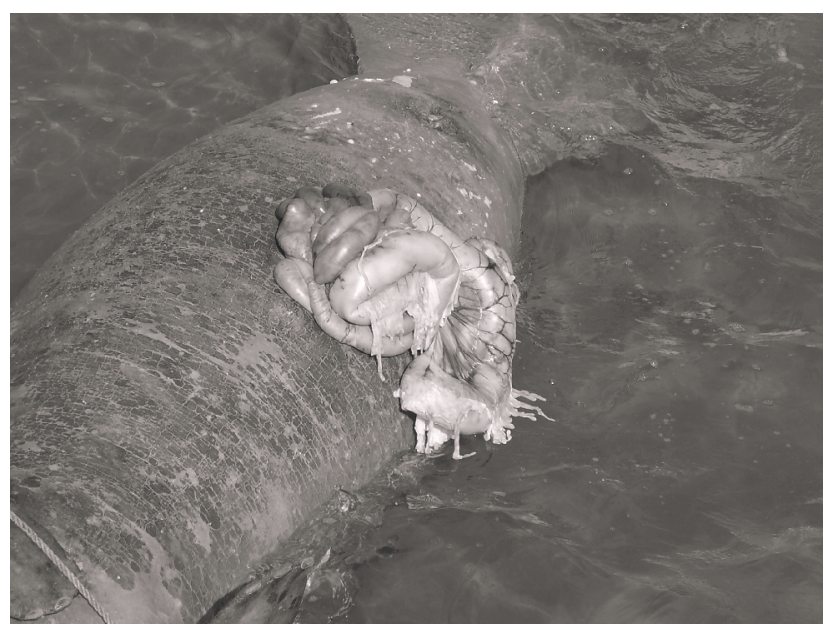

Figura 3. Manatíes encontrados en diferentes estados de descomposición en la costa de la Bahía de Nazabal. 